\section{Globulina Ligadora dos Hormônios Sexuais e Proteina Ligadora 1 do IGF-1: Marcadores para Resistência à Insulina na Pubarca Precoce?}

\begin{abstract}
RESUMO
A hiperinsulinemia parece contribuir para o hiperandrogenismo por reduzir os niveis séricos tanto da SHBG quanto da IGFBP-1. Avaliamos os níveis de SHBG e IGFBP-1 e sua correlação com androgênios e insulina em 44 meninas selecionadas com pubarca precoce (PP) e 18 controles

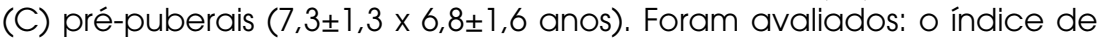
massa corporal (IMC), a idade óssea (IO) e os níveis séricos de SHBG, IGFBP-1, insulina (I), glicose $(G)$, testosterona $(T)$, androstenediona $(A)$, SDHEA e cortisol (F). Calculamos a relação glicose: insulina (G/I) no jejum como índice de resistência à insulina (RI). A IO foi maior na PP, mas o IMC foi semelhante aos C. Os niveis de SDHEA, T e A foram maiores, enquanto a SHBG e a IGFBP-1 foram menores na PP do que nos C. Na regressão simples, a SHBG mostrou correlação com IMC, F, SDHEA, T, I, G/I e IGFBP1, enquanto a IGFBP-1 se correlacionou com IMC, I e a G/l. No modelo de regressão múltipla, tanto a SHBG quanto a IGFBP-1 correlacionaramse apenas com o IMC e a taxa $G / /\left(r^{2}=0,45 ; p<0,01\right.$ e $r^{2}=0,44 ; p<0,01$, respectivamente). Nossos dados demonstram que o peso corporal e a insulina têm um papel sinérgico na regulação dos níveis da SHBG e da IGFBP-1, sugerindo que ambos podem ser marcadores sutis da RI na PP. (Arq Bras Endocrinol Metab 2003;47/3:261-265)
\end{abstract}

Descritores: SHBG; IGFBP-1; Resistência à Insulina; Pubarca Precoce.

\begin{abstract}
Sex Hormone Binding Globulin and IGF-Binding-Protein 1: Markers of Insulin Resistance in Premature Pubarche?

Hyperinsulinemia may contribute to hyperandrogenism because it reduces the levels of SHBG and IGFBP-1. In this study we determined serum levels of SHBG and IGFBP-1 and their association with androgen and insulin in 44 selected girls with premature pubarche (PP) and 18 prepubertal controls (C) $(7.3 \pm 1.3 \times 6.8 \pm 1.6 y e a r s)$. The body mass index (BMI) and bone age (BA) were determined, as well as the serum levels of SHBG, IGFBP-1, insulin (I), glucose (G), testosterone (T), DHEAS, androstenedione (A), 17-hydroxyprogesterone (17OHP) and cortisol (F). Fasting glucose to insulin ratio (G/I) was calculated as an index of insulin resistance (IR). BA was higher in PP than in C, but BMI was similar in both groups. Serum levels of DHEAS, $T$ and A were higher in PP than in $C$, whereas SHBG and IGFBP-1 were lower. SHBG was correlated with BMI, SDHEA, T, F, I, G/I and IGFBP-1; and IGFBP-1 was correlated with BMI, I and $G / I$ ratio. In the multiple regression model, SHBG and IGFBP-1 were correlated only with BMl and $G / /$ ratio $\left(r^{2}=0.45 ; p<0.01\right.$ and $r^{2}=0.44 ; p<0.01$, respectively). The present data demonstrate that body weight and insulin have a synergic role in the regulation of serum SHBG and IGFBP-1 levels, suggesting that both could be tenuous markers of IR in PP. (Arq Bras Endocrinol Metab 2003;47/3:261-265)
\end{abstract}

Keywords: SHBG; IGFBP-1; Insulin resistance; Premature pubarche.

\section{artigo original}

\author{
Rosimere J. Teixeiva \\ Denise Ginzbarg \\ Josele R. Freitas \\ Gabriela Fucks \\ Cristiane M. Silva \\ Maria Alice N. Bordallo
}

Unidade Docente Assistencial de
Endocrinologia, Hospital
Universitário Pedro
Ernesto (HUPE),
Universidade do Estado do
Rio de Janeiro (UERJ),
Rio de Janeiro, RJ.

Recebido em 25/02/03

Revisado em 30/05/03

Aceito em 04/0/06/03 
A PUbarCa PRECOCE (PP) é definida como o aparecimento de pêlos pubianos antes dos 8 anos nas meninas e 9 anos nos meninos $(1,2)$, sendo sua incidência quase 10 vezes maior nas meninas do que nos meninos (3). Na maioria dos casos a PP parece ser secundária a adrenarca precoce - maturação prematura ou exagerada da função normal da glândula adrenal $(2,3)$. Os androgênios adrenais, tais como a androstenediona (A), deidroepiandrosterona (DHEA), e especialmente o sulfato de DHEA (SDHEA) são moderadamente elevados para a idade cronológica, mas dentro da variação normal de acordo com os estádios puberais de Tanner para pêlos pubianos (2). A hiperplasia adrenal congênita nãoclássica (HACNC), em particular a deficiência da 21hidroxilase (2lOHD), é a segunda causa mais comum de PP, com uma freqüência que varia nas populações estudadas $(1,4)$.

$\mathrm{Na}$ ausência de defeito enzimático adrenal, a PP em meninas tem sido considerada desde há muito como uma variante normal do desenvolvimento puberal (5). Entretanto, o acompanhamento dessas meninas durante a adolescência tem revelado um aumento da incidência da síndrome dos ovários policísticos (SOP), uma forma de hiperandrogenismo ovariano funcional $(6,7)$, entidade associada a uma maior incidência de doença cardiovascular e diabetes mellitus na vida adulta.

A hiperinsulinemia e a resistência à insulina (RI) são características comuns em mulheres com hiperandrogenismo $(8,9)$. Além disso, a hiperinsulinemia pode contribuir diretamente para o hiperandrogenismo por reduzir os níveis tanto da SHBG quanto da IGFBP-1 (10-12). Nosso objetivo foi avaliar os níveis séricos da SHBG e da IGFBP-1 e sua correlação com os androgênios e a insulina na infância usando como modelo meninas com PP.

\section{CASUÍSTICA E MÉTODOS}

Foram selecionadas 44 meninas com PP devido a adrenarca precoce e 18 controles $(\mathrm{C})$ pré-puberais da mesma faixa etária $(7,3 \pm 1,3 \times 6,8 \pm 1,6$ anos, $p=N S)$.

Todas as participantes foram avaliadas quanto aos estádios puberais de Tanner para pêlos pubianos, estatura, peso, idade estatural (IE - percentil 50 do gráfico de crescimento) e idade óssea (IO - segundo os critérios de Greulich and Pyle). O índice de massa corporal [IMC - peso $(\mathrm{kg}) /$ estatura $\left(\mathrm{m}^{2}\right)$ ] foi calculado e considerado como obesidade quando foi maior ou igual ao percentil 95 (13).

Os níveis séricos de SHBG, IGFBP-1, testosterona (T), SDHEA, A, 17-hidroxiprogesterona

(17OHP), cortisol (F), insulina (I) e glicose $(\mathrm{G})$ foram medidos após 12 hs de jejum e dieta livre. $\mathrm{O}$ teste do ACTH foi realizado sempre que os níveis basais da $17 \mathrm{OHP}$ foram $\geq 150 \mathrm{ng} / \mathrm{dL}$ para descartar HACNC por 2lOHD (14). A taxa de jejum da glicose pela insulina $(G / I)$ foi calculada, sendo considerado como sugestivo de resistência à insulina (RI) um valor <7 (15).

Foram realizados os métodos $\mathrm{PAP}$ - enzimático colorimétrico para a dosagem de G; Immulite para as dosagens do SDHEA, F e SHBG; e RIE para as dosagens de T, A, 17OHP e I; e imunoradiométrico para a dosagem de IGFBP-1. Os valores dos coeficientes intra e inter-ensaio foram respectivamente de 7,6 e $15 \%$ para SDHEA; 7,4 e $7,7 \%$ para $\mathrm{T} ; 4,3 \%$ e $4,7 \%$ para A; 3,5 e $8,5 \%$ para $17 \mathrm{OHP} ; 7,0$ e $10,3 \%$ para F; 3,0 e $8,0 \%$ para SHBG; 4,6 e 6,0\% para IGFBP-1; e 8,4 e $10 \%$ para I.

Para a análise estatística foram realizados os testes $t$ de Student ou de Wilcoxon. Para as variáveis categóricas foi utilizado o teste não-paramétrico Quiquadrado, procedendo-se à correção de Yates nos casos indicados. A correlação linear simples de Pearson ou Sperman foi usada para descrever a associação entre androgênios, F, G, I, taxa G/I e IMC com SHBG e IGFBP-1. Para as variáveis que apresentaram $\mathrm{p}<0,05$, a regressão linear múltipla - forward, stepwise ou backward - foi realizada para identificar os modelos que melhor descrevessem as variações nos níveis da SHBG e IGFBP-1. Para cada variável foi dado um coeficiente $\beta$, seu desvio-padrão (SD) e correspondente significância no final do modelo. Os resultados foram expressos em média $\pm \mathrm{SD}$, e o valor de $\mathrm{p}<0,05$ foi considerado estatisticamente significativo.

Trata-se de um estudo descritivo e analítico de corte transversal. O protocolo do estudo foi aprovado pelo Comitê de Ética em Pesquisa do HUPE. Foi solicitado aos pais e/ou responsáveis o consentimento por escrito, após informação prévia, para a inclusão das meninas no estudo.

\section{RESULTADOS}

A IO $(8,2 \pm 1,8 \times 6,5 \pm 2,2$ anos, $\mathrm{p}=0,01)$ e a IE $(8,3 \pm 1,5 \times 7,4 \pm 1,8$ anos, $\mathrm{p}=0,04)$ foram maiores na $\mathrm{PP}$, mas o IMC $\left(19,1 \pm 3,7 \times 19,1 \pm 4,9 \mathrm{~kg} / \mathrm{m}^{2}\right)$ foi semelhante aos C. Não houve diferença entre os grupos quanto à proporção de casos com obesidade (41\% x $33 \%, \mathrm{p}=\mathrm{NS}$ ).

Os níveis séricos de SDHEA $(0,61 \pm 0,4 \mathrm{x}$ $0,27 \pm 0,23 \mu \mathrm{g} / \mathrm{mL}, \quad \mathrm{p}<0,0008), \mathrm{T}(83,4 \pm 60,4 \quad \mathrm{x}$ $56,2 \pm 25,3 \mathrm{pg} / \mathrm{mL}, \mathrm{p}=0,003)$ e $\mathrm{A}(704,5 \pm 383,3 \times$ 
$387,5 \pm 208,8 \mathrm{pg} / \mathrm{mL}, \mathrm{p}=0,003$ ) foram maiores na PP do que nos C. Não houve diferença entre os grupos quanto à $\mathrm{G}(84,0 \pm 9,4 \times 88,0 \pm 11,6 \mathrm{mg} / \mathrm{dL})$, I $(11,3 \pm 6,7 \quad x \quad 11,3 \pm 6,9 \mu \mathrm{IU} / \mathrm{mL}), \quad$ taxa $\mathrm{G} / \mathrm{I}$ $(12,3 \pm 14,2 \times 11,9 \pm 8,8)$ e $\mathrm{F}(12,8 \pm 5,6 \times 11,7 \pm 5,7$ $\mu \mathrm{g} / \mathrm{dL})$. Entretanto, a SHBG $(75,6 \pm 43,8 \times 90,8 \pm 32,5$ $\mathrm{nmol} / \mathrm{L}, \quad \mathrm{p}=0,02)$ e a IGFBP-1 $(63,4 \pm 38,4 \mathrm{x}$ $85,7 \pm 56,5 \mathrm{ng} / \mathrm{mL}, \mathrm{p}=0,08)$ foram menores na PP do que nos C (gráfico $\mathrm{l}$ ).

$\mathrm{Na}$ regressão simples, a SHBG mostrou correlação com IMC $(r=0,54 ; \mathrm{p}<0,001)$, SDHEA $(r=-0,30$; $\mathrm{p}=0,02), \mathrm{T}(\mathrm{r}=0,24 ; \mathrm{p}=0,07), \mathrm{F}(\mathrm{r}=0,33 ; \mathrm{p}=0,01), \mathrm{I}$ $(\mathrm{r}=-0,31 ; \mathrm{p}=0,02)$, taxa $\mathrm{G} / \mathrm{I}(\mathrm{r}=0,34 ; \mathrm{p}=0,01)$ e IGFBP-1 $(r=0,42 ; \mathrm{p}=0,001)$, enquanto a IGFBP- 1 se correlacionou com IMC $(\mathrm{r}=0,64 ; \mathrm{p}<0,001)$, I $(\mathrm{r}=-$ $0,49 ; \mathrm{p}<0,001)$ e a taxa $\mathrm{G} / \mathrm{I}(\mathrm{r}=-0,47 ; \mathrm{p}<0,001)$. Entretanto, no modelo de regressão múltipla o IMC e a taxa $\mathrm{G} / \mathrm{I}$ foram variáveis independentes tanto para a SHBG quanto para a IGFBP-1 $\left(\mathrm{r}^{2}=0,45 ; \mathrm{p}<0,01 \mathrm{e}\right.$ $\mathrm{r}^{2}=0,44 ; \mathrm{p}<0,01$, respectivamente) (tabela $\mathrm{l}$ ).

\section{DISCUSSĀO}

O aumento dos níveis de insulina tem sido considerado responsável pelo desenvolvimento de hiperandrogenismo adrenal e ovariano (16). Adicionalmente, tanto a insulina como o fator de crescimento 1 semelhante à insulina (IGF-1) são capazes de estimular a produção de androgênios pelas células da teca e intersticial dos ovários assim como aumentar a esteroidogênese e a responsividade ao ACTH de células adrenocorticais humanas em cultura $(17,18)$. A insulina também modula a ação do IGF-1 e da IGFBP-1 e inibe a produção da SHBG em linhagens de células do hepatoma humano (19). Adicionalmente, tem sido demonstrado que em mulheres hiperandrogênicas a diminuição dos níveis de insulina através de agentes sensibilizadores da insulina reduz os níveis séricos dos androgênios, aumenta a concentração da SHBG e restaura a ovulação e os ciclos menstruais, provavelmente através da redução da atividade do citocromo P450c17 $\alpha$ ovariano (20).

\begin{tabular}{|c|c|c|c|c|c|}
\hline \multirow{2}{*}{$\begin{array}{c}\begin{array}{c}\text { Variável } \\
\text { Dependente }\end{array} \\
\text { SHBG }\end{array}$} & \multicolumn{2}{|c|}{$\begin{array}{l}\text { Correlação } \\
\text { simples (r) }\end{array}$} & \multicolumn{3}{|c|}{$\begin{array}{c}\text { Regressão múltipla } \\
\beta \quad S D \quad r^{2}\end{array}$} \\
\hline & $\begin{array}{c}\text { IMC } \\
\operatorname{taxa} G / \mid\end{array}$ & $\begin{array}{c}-0,54^{*} \\
0,34^{\star *} \\
-0,31 \#\end{array}$ & $\begin{array}{l}-0,48^{\star} \\
0,37^{\star *}\end{array}$ & $\begin{array}{l}0,13 \\
0,15\end{array}$ & 0,45 \\
\hline |GFBP-1 & $\begin{array}{c}\text { IMC } \\
\operatorname{taxa} G / \mid \\
\mid\end{array}$ & $\begin{array}{c}-0,64^{*} \\
0,47^{\star} \\
-0,49^{*}\end{array}$ & $\begin{array}{l}-0,31^{* *} \\
0,35^{\star *}\end{array}$ & $\begin{array}{l}0,12 \\
0,14\end{array}$ & 0,44 \\
\hline
\end{tabular}

SHBG: globulina ligadora dos hormônios sexuais; IGFBP-1: proteína ligadora 1 do IGF-l; IMC: índice de massa corporal; G: Glicose; I: Insulina. ${ }^{*} \mathrm{p}<0,001 ;{ }^{* *} \mathrm{p}<0,01 ; \# \mathrm{p}=0,02$

A. SHBG $(\mathbf{n m o l} / \mathbf{L})$

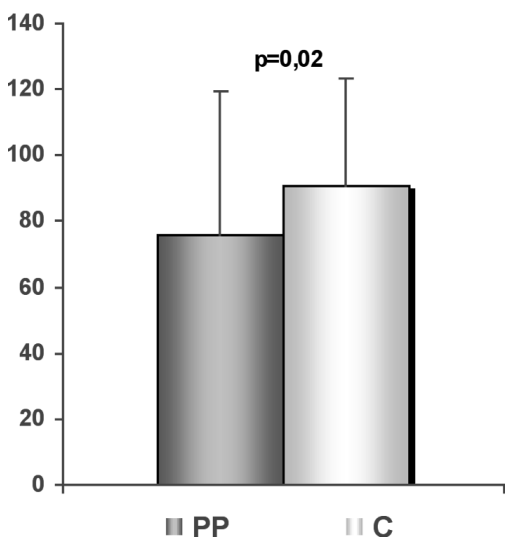

B. IGFBP-1 (ng/mL)

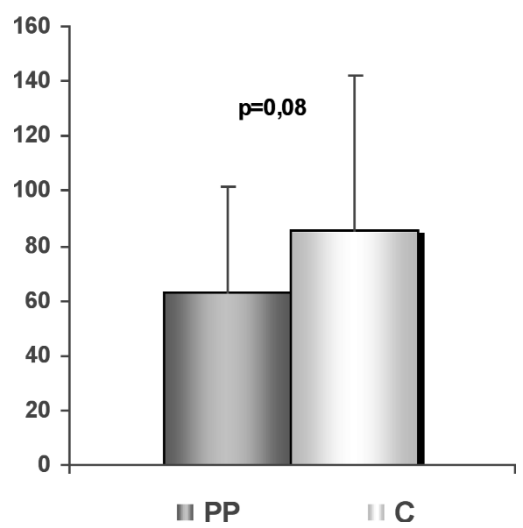

Gráfico 1. A- Niveis séricos da globulina ligadora dos hormônios sexuais (SHBG) em meninas com pubarca precoce (PP) e controles pré-puberais (C); B- niveis séricos da proteína ligadora 1 do IGF-I (IGFBP-1) em meninas com PP e C. 
A prevalência de hiperinsulinemia, hiperandrogenismo ovariano, disfunção ovulatória e dislipidemia pode estar aumentada em adolescentes com história prévia de PP (21). Por sua vez, as meninas com PP parecem apresentar hiperinsulinismo e uma relação de jejum da insulina/glicose (FIGR - Fasting Insulin to Glucose Ratio) sugestiva de RI desde antes da puberdade, quando a PP é diagnosticada, e através de todos os estádios do desenvolvimento puberal $(12,22,23)$. Em estudo prévio, demonstramos que o hiperandrogenismo adrenal pode não ter um efeito adverso sobre a sensibilidade à insulina (SI) na infância, contudo a presença de obesidade, acanthosis nigricans e da FIGR sugestiva de RI são relativamente comuns em meninas com PP (22).

Neste estudo, não encontramos diferença entre os níveis de insulina e da taxa $\mathrm{G} / \mathrm{I}$ entre as meninas com PP e controles. Devemos considerar que a baixa idade de nossas meninas, assim como o método utilizado e o IMC elevado em ambos os grupos, podem ter interferido na avaliação do nível sérico da insulina. Embora Legro e cols. (24) considerem que a taxa G/I seja de fácil obtenção, altamente sensível e específica para a medida da SI, sabemos que as anormalidades na ação da insulina podem ser pobremente detectadas através das dosagens de jejum (25). Entretanto, os testes mais precisos são mais demorados, trabalhosos, de alto custo e não são fáceis de realizar em grande escala, principalmente em crianças, por motivos éticos (24).

A hiperinsulinemia é usualmente acompanhada pela diminuição dos níveis da SHBG e da IGFBP-1 em mulheres com a SOP $(10,11,26)$ e em meninas pré e pós-puberais com PP (12), embora na PP esses achados não sejam unânimes (27). Nesta casuística, encontramos níveis reduzidos tanto da SHBG quanto da IGFBP-1 na PP apesar da ausência de hiperinsulinemia comparandose ao grupo C. Em crianças com PP $(12,28)$, adolescentes com hiperandrogenismo (8) e crianças obesas (29) tem sido descrita uma forte correlação entre insulina e SHBG. Em geral, é aceito que a síntese da SHBG seja principalmente regulada pelos níveis dos androgênios (30). Em meninas com PP, a redução dos níveis da SHBG parece se correlacionar mais com o peso corporal do que com a $\mathrm{T}$, a diidrotestosterona e os androgênios adrenais (28). Em estudo recente, avaliando meninas com PP e controles pré-puberais, descrevemos que o $3 \alpha$-diolG também pode ser responsável pela regulação da síntese da SHBG na infância (31). Estes fatos foram respaldados neste estudo, visto que o índice de RI e IMC comportaram-se como variáveis independentes dos androgênios para a SHBG.

Meninas com PP apresentam correlação inversa entre os níveis da SHBG e IGF-1 enquanto a IGFBP-1 segue o mesmo padrão e é fortemente correlacionada com a SHBG (32). Nesta amostra a IGFBP-l se correlacionou com o índice de RI independentemente dos níveis da insulina e SHBG. A modulação dos níveis da IGFBP-1 pela insulina pode resultar em aumento da relação IGF-1/IGFBP-1 e como conseqüência aumentar o "IGF-1 livre" e a sensibilidade tecidual ao IGF-1, que pode ser um potente indutor da atividade da $5 \alpha$-redutase, que por sua vez parece ser dependente dos androgênios (33).

Nossos dados demonstram que o peso corporal e a insulina têm um papel sinérgico na regulação tanto dos níveis da SHBG quanto da IGFBP-1, sugerindo que ambos podem ser marcadores sutis da RI na PP. Como a regulação dos níveis da SHBG e da IGFBP-1 pela insulina é um fenômeno fisiológico generalizado, defendemos a idéia de que estas proteínas podem ser marcadores úteis dos estados de RI, desde que exista uma secreção endógena intacta.

A insulina é um hormônio aterogênico e constitui um fator de risco independente para doença cardiovascular e síndrome plurimetabólica na vida adulta (34). Logo, a presença de níveis reduzidos da SHBG e da IGFBP-1 e sua associação com resistência à insulina indicam que as meninas com $\mathrm{PP}$ podem apresentar desde a infância um maior risco para o desenvolvimento dessas desordens.

\section{REFERÊNCIAS}

1. Siegel SF, Finegold DN, Urban MD, McVie R, Lee PA. Premature pubarche: etiological heterogeneity. J Clin Endocrinol Metab 1992;74:239-47.

2. Rosenfield RL. Normal and almost normal precocious variations in pubertal development premature pubarche and premature thelarche revisited. Horm Res 1994:41:7-13

3. Reiter EO, Saenger P. Premature adrenarche. The Endocrinologist 1997;7:85-8.

4. Ibáñez L, Bonnin MR, Zampolli M, Prat N, Alia PJ, Navarro MA. Usefulness of an ACTH test in the diagnosis of nonclassical 21-hydroxylase deficiency among children presenting with premature pubarche. Horm Res 1995;44:51-6.

5. Voutilainen R, Perheentupa J, Apter D. Benign premature adrenarche: clinical factures and serum steroid levels. Acta Paediatr Scand 1983;72:707-11.

6. Ibáñez L, Potau N, Virdis R, Zampolli M, Terzi C, Miquel G, et al. Postpubertal outcome in girls diagnosed of premature pubarche during childhood: increased frequency of functional ovarian hyperandrogenism. J Clin Endocrinol Metab 1993;76:1599-603.

7. Apter D. How possible is the prevention of polycystic ovary syndrome development in adolescent patients with early onset of hyperandrogenism. J Endocrinol Invest 1998;21:613-7.

Arq Bras Endocrinol Metab vol $47 n^{\circ} 3$ Junho 2003 
8. Ibáñez L, Potau N, Georgopoulos N, Prat N, Gussinyé M, Carrascosa A. Growth hormone, insulin-like growth factor-1 axis and insulin secretion in hyperandrogenic adolescents. Fertil Steril 1995;64:1113-9.

9. Mauras N, Welch S, Rini A, Haymond MW. Ovarian hyperandrogenism is associated with insulin resistance to both peripheral carbohydrate and whole-body protein metabolism in post-pubertal young females: a metabolic study. J Clin Endocrinol Metab 1998;83:1900-5.

10. Nestler JE, Powers LP, Matt DW. A direct effect of hyperinsulinemia on serum sex hormone-binding globulin levels in obese women with the polycystic ovary syndrome. $\mathrm{J}$ Clin Endocrinol Metab 1991;72:83-9.

11. Peiris AN, Stagner Jl, Plymate SR, Vogel RL, Heck M, Samols E. Relationship of insulin secretory pulses to sex hormone-binding globulin levels in normal men. J Clin Endocrinol Metab 1993;76:279-82.

12. Ibáñez L, Potau N, Zampolli M, Riqué S, Saenger P, Carrascosa $A$. Hyperinsulinemia and decreased insulin-like growth factor-binding protein-1 are common features in prepubertal and pubertal girls with a history of premature pubarche. J Clin Endocrinol Metab 1997;82:2283-8.

13. Schonnfeld-Warden N, Warden $\mathrm{CH}$. Obesidade pediátrica: uma visão global da etiologia e tratamento. In: Styne DM, redator convidado. Clínicas Pediátricas da América do Norte. Rio de Janeiro: Interlivros: 1997.p.343-66.

14. Pang S. Congenital adrenal hyperplasia. Baillieres Clin Obstet Gynaecol 1997;11:281-306.

15. DiMartino-Nardi J. Premature adrenarche: findings in prepubertal African-American and Caribbean-Hispanic girls. Acta Paediatr 1999;433(Suppl):67-72

16. Rosenfield RL. Ovarian and adrenal function in polycystic ovary syndrome. Endocrinol Metab Clin North Am 1999:28:265-93.

17. Cara JF, Rosenfield RL. Insulin-like growth factor-I and insulin potentiate luteinizing hormone induced androgen synthesis by rat ovarian thecal-interstitial cells. Endocrinology 1988; $123: 733-9$

18. Lálleman D, Penhoat A, Lebrethon MC, Ardèvol R, Baehr V, Oelkers W, et al. Insulin-like growth factor enhance steroidogenic enzyme and corticotropin receptor messenger ribonucleic acid levels and corticotropin steroidogenic responsiveness in cultured human adrenocortical cells. J Clin Endocrinol Metab 1996;81:3892-7.

19. Suikkari AM, Koivisto VA, Rutanen EM, Yki-Järvinem $H$ Karonen SL, Sépala M. Insulin regulates the serum levels of low molecular weight insulin-like growth factor-binding protein. J Clin Endocrinol Metab 1988;66:266-72.

20. Dunaif A, Scott D, Finegood D, Quintana B, Whitcomb R. The insulin sensitising agent troglitazone improves metabolic and reproductive abnormalities in the polycystic ovary syndrome. J Clin Endocrinol Metab 1996;81:3299-306.

21. Ibáñez L, Potau N, Zegher F. Precocious pubarche in girls may be a marker of a polyendocrinopathy. In: Bourguignon J-P, Plant TM, eds. The onset of puberty in perspective. Amsterdam: Elsevier; 2000.p.289-97.

22. Teixeira RJ, Gazolla HM, Cunha SB, Bordallo MAN, Guimarães MM. Resistência à insulina na pubarca precoce - Relação com os androgênios. Arq Bras Endocrinol Metab 2001;45:278-84.
23. Teixeira RJ, Silva VCG, Freitas JR, Dimetz T, Cunha SB, Guimarães MM. The relationship between ovarian structure and hyperandrogenism in premature pubarche. $\mathbf{J}$ Pediatr Endocrinol Metab 2001;14:257-65.

24. Legro RS, Finegood, Dunaif A. A fasting glucose to insulin ratio is a useful measure of insulin sensitivity in women with polycistic ovary syndrome. J Clin Endocrinol Metab 1998;83:2694-7.

25. American Diabetic Association. Consensus development conference on insulin resistance. Diabetes Care 1998:21:310-4.

26. Nobels F, Dewailly D. Puberty and polycistic ovarian syndrome: the insulin/insulin-like growth factor I hypothesis. Fertil Steril 1992;58:655-66.

27. Vuguin $\mathrm{P}$, Wasserman $\mathrm{E}$, Linder $\mathrm{B}$, Oppenheimer $\mathrm{E}$, Rosenfeld $R$, Saenger $P$, et al. The role of insulin sensitivity, IGF-I, IGFBP-1 and IGFBP-3 in the hyperandrogenism in black and hispanic girls with premature adrenarche. Horm Res 1997;48(suppl 2): 104.

28. Balducci R, Finocchi $G$, Mangiantini A, Maggi $C$, Bianchi P, Guglielmi R, et al. Lack of correlation between sex hormone-binding globulin, adrenal and peripheral androgens in precocious adrenarche. J Endocrinol Invest 1992; 15:501-5.

29. Gascon F, Valle M, Martos R, Ruz FJ, Rios R, Montilla P, et al. Sex hormone-binding globulin as a marker for hyperinsulinemia and/or insulin resistance in obese children. Eur J Endocrinol 2000; 143:85-9.

30. Hautanen A. Synthesis and regulation of sex hormonebinding globulin in obesity. Int J Obes Relat Metab Disord 2000;24:64-70.

31. Teixeira RJ, Dimetz T, Bordallo MAN, Guimarães MM. Papel dos androgênios adrenais e periféricos na modulação dos níveis da globulina ligadora dos hormônios sexuais na pubarca precoce. Arq Bras Endocrinol Metab 2002;46:520-5.

32. Teixeira RJ, Silva VCG, Gazolla HM, Cunha SB, Guimarães MM. The relationship between ovarian structure and serum insulin, insulin-like growth factor-I (IGF-I) and its binding protein (IGFBP-1 and IGFBP-3) levels in premature pubarche. J Pediatr Endocrinol Metab 2002; 15:69-75.

33. Toscano V, Bianchi P, Balducci R, Guglielmi R, Mangiantini $A$, Lubrano $C$, et al. Lack of linear relationship between hyperinsulinaemia and hyperandrogenism. Clin Endocrinol 1992;36:197-202.

34. Orchard TJ, Becker DJ, Bates M, Kuller LH, Drash AL. Plasma insulin and lipoprotein concentrations: an atherogenic association? Am J Epidemiol 1983; 1 18:326-37.

\section{Endereço para corespondência:}

Rosimere de Jesus Teixeira Rua Silva Rabelo 10-307 Méier 20735-080 Rio de Janeiro, RJ

FAX: (021) 2587-6447

e-mail: rosijt@fst.com.br 